Article

\title{
Development of a Thulium Fiber Laser for an Atomic Spectroscopy Experiment
}

\author{
Ronnie Currey *, Ali Khademian and David Shiner \\ Department of Physics, University of North Texas, Denton, TX 76203, USA; alikhademian@my.unt.edu (A.K.); \\ shiner@unt.edu (D.S.) \\ * Correspondence: ronniecurrey@my.unt.edu
}

Received: 20 December 2019; Accepted: 9 February 2020; Published: 15 February 2020

\begin{abstract}
A convenient thulium fiber laser source is described with $3 \mathrm{~W}$ of output power operating at a wavelength of $2059 \mathrm{~nm}$ with a slope efficiency of $49 \%$ with respect to input pump power and $60 \%$ with respect to absorbed pump power. The laser was applied in an atomic helium spectroscopy experiment to quench ${ }^{3} \mathrm{He}(2058.63 \mathrm{~nm})$ and ${ }^{4} \mathrm{He}(2058.69 \mathrm{~nm})$ meta-stable singlets $\left(2^{1} \mathrm{~S}_{0}\right)$, allowing for further investigation of the helium fine structure. The customized laser effectively eliminates the singlet counts to well below a background level (1\%). A simplified analysis describes the basic laser performance with fitted constants in reasonable agreement with previous work.
\end{abstract}

Keywords: fiber; laser; fiber laser; thulium; thulium fiber laser; optics; rare-earth elements; spectroscopy

\section{Introduction}

The key benefits of double clad single transverse mode fiber lasers have been well established, such as efficient and powerful pumping, alignment ease and stability, good heat dissipation due to large surface area to volume ratio, and excellent beam quality [1,2]. For spectroscopic applications, small diameters (typically $250 \mu \mathrm{m}$ ) and long fiber lengths allow for both compact designs and dense mode spacing to conveniently excite very narrow spectral lines. Fiber Bragg gratings are frequently employed to create the laser cavity inside the fiber and select the necessary wavelength and bandwidth. Grating centers can be tuned with temperature for fine control of output wavelength $(\approx 0.02 \mathrm{~nm} / \mathrm{C}$ for $\approx 2 \mu \mathrm{m})$.

Silica (Si) with rare-earth (RE) do-pants provide very broad homogeneous fluorescent emission. When placed in a silica glass host, thulium (Tm) has several different absorption bands, located at $790 \mathrm{~nm}, 1210 \mathrm{~nm}$, and $1670 \mathrm{~nm}[3,4]$. The emission spectrum for the ${ }^{3} \mathrm{~F}_{4}{ }^{-} \mathrm{H}_{6}$ transition spans about 400 $\mathrm{nm}$, ranging between $1700 \mathrm{~nm}$ to $2100 \mathrm{~nm}$ [5]. This emission range extends into the eye-safe regime and has many applications such as laser detection and ranging (LIDAR) for wind shear detection radar, remote sensing, atmospheric pollution monitoring, surgery, range finders, as well as for inducing transitions in atomic spectroscopy experiments [6].

Fiber coupled laser diodes emitting around $790 \mathrm{~nm}$ are capable of generating high signal powers especially when employing cladding pumped schemes [7-9].Thulium can be pumped at any of the wavelength bands shown in Figure 1, but the $790 \mathrm{~nm}$ band is of particular interest because at high doping $(\approx 4 \%-6 \%$ ) it yields a near 2 for 1 cross relaxation process populating the upper lasing level, where the cross relaxation efficiency can be defined as the average number of lasing levels created per pump photon absorbed and increases the overall efficiency that would otherwise be limited to the usual quantum defect between lasing and pump frequencies [10]. Efficiencies have been reported to exceed $60 \%[3,11]$. 


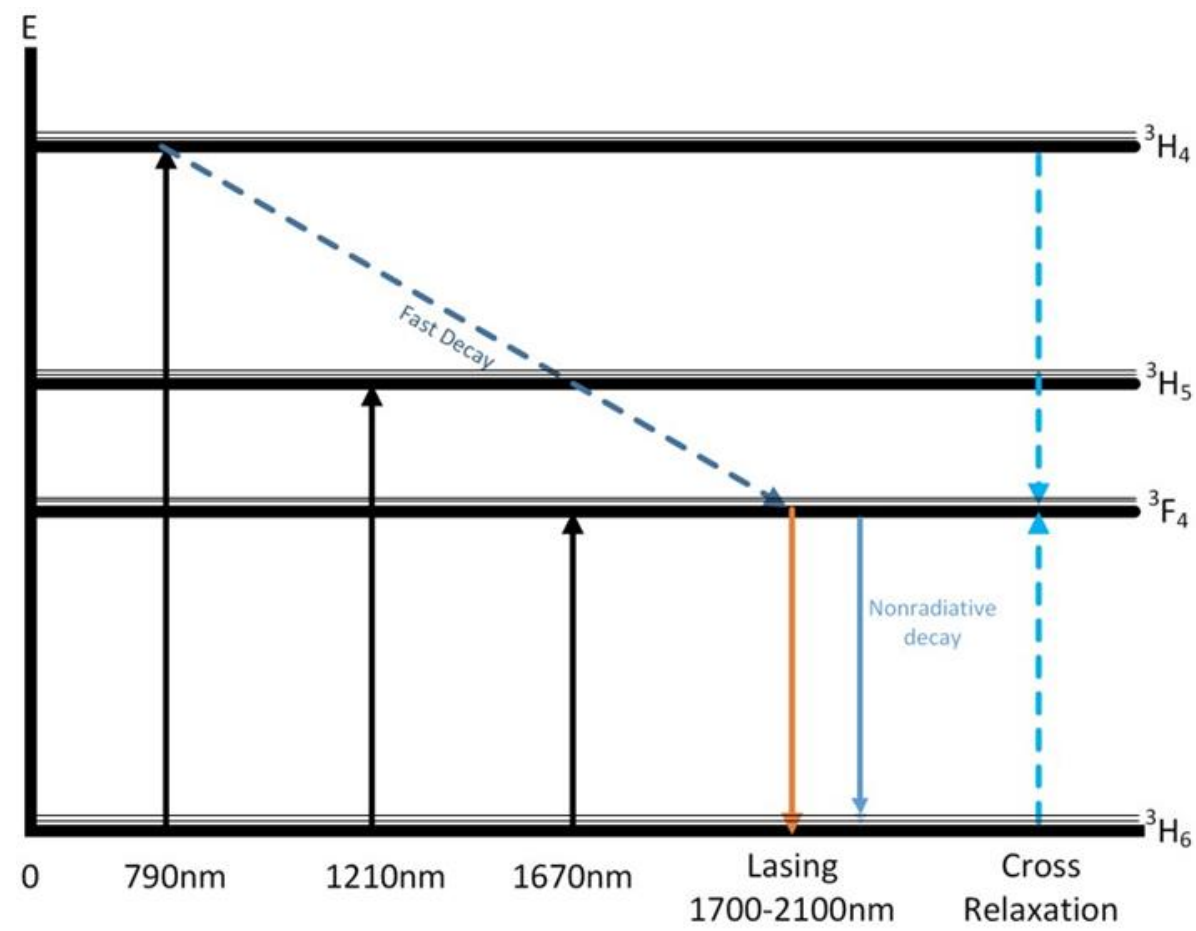

Figure 1. Energy Levels of Thulium.

\section{Materials and Methods}

\subsection{Laser Design}

The laser described in this report is pumped with a diode module centered at $793 \mathrm{~nm}$ with a maximum output power of $8 \mathrm{~W}$ (BWT Beijing), see Figure 2. This pump laser is fiber coupled with $105 \mu \mathrm{m} / 125 \mu \mathrm{m}$ NA $=0.15$ fiber and has a bandwidth of $3 \mathrm{~nm}$. It also is built with feedback protection between 1900-2000 nm. The diode module is fused to a $(2+1) \times 1$ multi-mode pump combiner (ITF Technologies) to facilitate launching the pump power into the active fiber. For this experiment, only one of the combiner's input ports was used. The input was designed to match the pump laser fiber and so had $105 \mu \mathrm{m} / 125 \mu \mathrm{m} \mathrm{NA}=0.15$ fiber on the input pump side. The signal side had $10 \mu \mathrm{m} / 125 \mu \mathrm{m}$ low index double clad NA $=0.46$ fiber to match the active fiber. We measured $95 \%$ pump beam combiner efficiency and 99\% transmission efficiency between the combiner signal ports (called $T_{\mathrm{c}}$ below). Launching the pump power from the combiner into the $10 \mu \mathrm{m} / 130 \mu \mathrm{m}$ low index Tm active fiber was more difficult than we had expected. The $\approx 3 \mathrm{~cm}$ of low index recoat was determined to be responsible for most of the coupling loss. With improvements in cleanliness and technique, the loss was reduced to $5 \%$, as verified by cutback experiments. Nevertheless, simple observation with an optical microscope indicates the index uniformity of the recoated fiber was not nearly as uniform as the commercial fiber. The active fiber consists of $2.15 \mathrm{~m}$ of thulium doped $10 \mu \mathrm{m} / 130 \mu \mathrm{m}$ low index $\mathrm{NA}=0.46$ fiber (Nufern, East Granby, CT, USA) designed to give a truly single-mode profile $\left(\mathrm{TEM}_{00}\right)$. The thulium fiber has a cladding absorption of $4.6 \mathrm{~dB} / \mathrm{m}$ at $793 \mathrm{~nm}$. This fiber is expected to absorb nominally $90 \%$ of the pump light launched into the gain fiber (7\% was measured exiting the back which is consistent with a $90 \%$ absorption and the subsequent losses through grating and recoat sections). The gain length was chosen basically by balancing the cost of lost pump photons versus the cost of additional Tm fiber. The frequency mode spacing of the overall cavity length $(\mathrm{c} / 2 \mathrm{~nL}=150 \mathrm{MHz}) \mathrm{was}$ already dense enough to overlap the broad spectral feature (300 MHz FWHM), so no additional length was needed. The absorbed pump power $P_{\mathrm{abs}}$ in terms of the diode power $P_{\text {diode }}$ is given by:

$$
P_{\text {abs }}=\eta_{\mathrm{p}} * P_{\text {diode }}
$$


with an absorption efficiency $\eta_{\mathrm{p}}$ of $81 \%$, given by the product of the beam combiner (0.95), launch (0.95), and absorption (0.90) efficiencies.

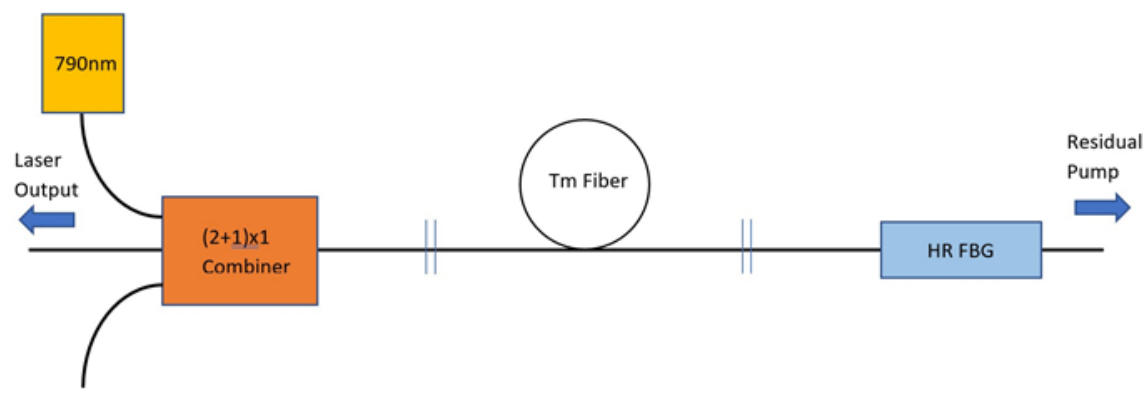

Figure 2. Thulium Fiber Laser Schematic.

The laser cavity is created by employing a highly reflective fiber Bragg gating $\left(R_{1} \approx 99.7 \%\right.$ inferred from an optical spectrum analyzer measurement of $T_{1}=0.3 \%$ as supplied by the vendor, $\mathrm{O} / \mathrm{E}$ Land). It is centered at $2058.45 \mathrm{~nm}$ with a bandwidth of $1.2 \mathrm{~nm}$. The grating is fused to the back end of the active fiber and the Fresnel reflection of $R_{2}=3.3 \%$ forms the output coupler at the front end. The fiber Bragg grating (FBG) was designed to insure the laser bandwidth easily overlapped the required transitions even allowing for large grating centering errors and without any grating temperature control.

\subsection{Atomic Helium Beam}

The above laser was designed for use in a high precision measurement of fine and hyper-fine structure in the stable isotopes $\left({ }^{3} \mathrm{He}\right.$ and $\left.{ }^{4} \mathrm{He}\right)$ of the helium atom. Metastable helium is created through collisional excitation with electrons produced by thermionic emission from a tungsten filament, see Figure 3. This produces approximately equal sublevel populations in the singlet and triplet states.

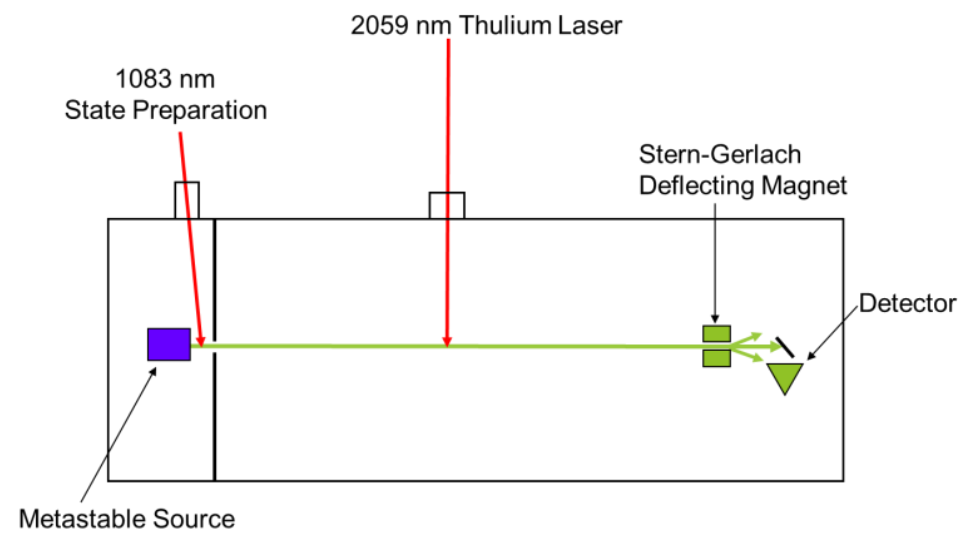

Figure 3. Helium Atomic Experiment.

The atomic beam is collimated through slits, approximately 4 mils wide, to reduce Doppler broadening. The above laser and a $1083 \mathrm{~nm}$ laser depopulate the singlet and the $\mathrm{m}=0$ magnetic sublevel respectively. Separating the $m=0,+1$, and -1 sublevels is done with a set of Stern-Gerlach magnets, which allows for selective detection of the $m=0$ state via electron ejection from a gold plated grazing surface (surface Penning ionization or Auger deexcitation). The resulted electrons emit and are collected by a continuous electron multiplier. A signal is detected by using a spectroscopic modulated laser of $1083 \mathrm{~nm}$ to allow for observations of the $\mathrm{J}=0,1,2$ states and the comparison of the spacing of these levels allow for measurements of the fine and hyper-fine structure. The remaining gas is circulated through a liquid nitrogen dewar to remove background gas and to reuse the rare and expensive ${ }^{3} \mathrm{He}$. 


\section{Results}

\subsection{Laser Characterization}

Upon testing the laser, the threshold was found to be $1.7 \mathrm{~W}$ with a bandwidth of $70 \mathrm{GHz}\left(50{ }^{\circ} \mathrm{C}\right)$. The laser produces an output of over $3 \mathrm{~W}$ corresponding to a slope efficiency of $60 \%$ with respect to absorbed power and follows a TEM 00 profile.

The laser shows a fairly abrupt threshold and a fairly uniform slope efficiency, see Figure 4. Under such conditions a simplified analysis may be adequate to determine if the laser is operating as expected, see for example [12]. It is important to point out that more detailed and complete laser models have been developed and reported in literature [4,9], and they help motivate the simplifications taken below. To estimate the slope efficiency, a homogeneously broadened lasing transition is assumed with no parasitic pump or laser absorption, no excited state absorption, hole burning, etc. In steady state, any attempt at additional population in the lasing level above threshold results in a shortened lifetime through stimulated emission. Hence, the slope efficiency $\eta_{\text {slope }}$ is written as

$$
\eta_{\text {slope }}=\frac{h v_{\text {laser }}}{h v \text { pump }}(C R) \eta_{\text {ex }}
$$

Laser Output Vs Absorbed Input

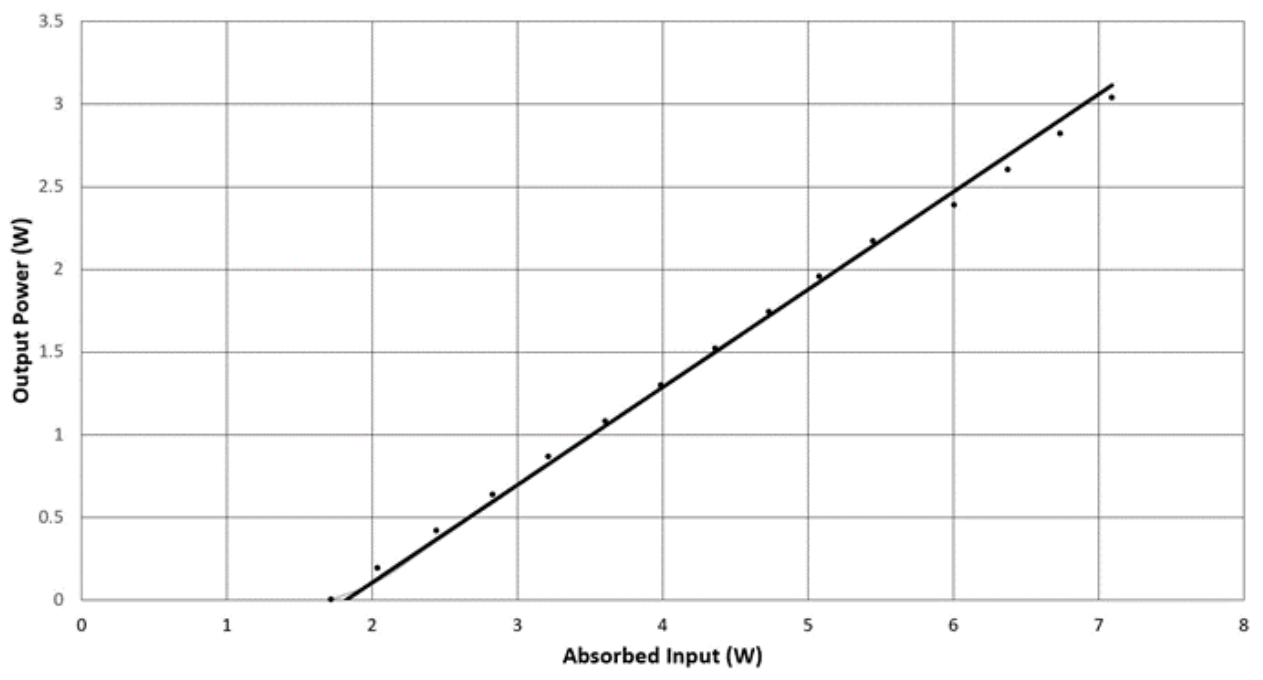

Figure 4. Laser Output vs. Absorbed Input.

In addition to the cross relaxation efficiency (CR) and the quantum defect, the extraction efficiency $\left(\eta_{\text {ex }}\right)$ indicates the fraction of the stimulated photons that exit the cavity and form the laser output. Because the output coupling of $96.7 \%$ is so large, the additional internal $2.4 \%$ loss has only a small effect of laser operation (note the nonuniform laser intensity within the cavity reduces sensitivity to losses from the back side of the laser in contrast to the threshold estimate below). With $\eta_{\mathrm{ex}}=0.974$, the CR term, which was not independently determined, was simply adjusted to 1.6 to yield the measured slope efficiency. This value of 1.6 is composition dependent (particularly the $\mathrm{Al}$ and Tm concentrations) [7], and is less than the best reported values of approximately $1.8[3,9]$.

The threshold of the laser occurs when the round trip gain $\mathrm{G}$ from inversion equals the round trip losses. The gain is found using the absorption $\left(\sigma_{\mathrm{a}}=2.8 \times 10^{-28} \mathrm{~m}^{2}\right)$ and emission $\left(\sigma_{\mathrm{e}}=9 \times 10^{-26} \mathrm{~m}^{2}\right)$ cross sections at $2059 \mathrm{~nm}$ taken from published data on similar fibers [13-16]. The data agreed to $\pm 10 \%$. The variation could be partially attributed to composition dependence. The value for $\sigma_{\mathrm{a}}$ was verified to within $7 \%$ by measuring the fiber absorption at $2059 \mathrm{~nm}$ (assuming negligible parasitic absorption). Also the modal overlap with the core $(\Gamma=0.75)$, the fiber core area $\mathrm{A}$, and the Tm concentration density 
$\left(3 \times 10^{26} \mathrm{~m}^{3}\right)$ are required. To good approximation, the population is primarily only in the ground and lasing levels, $n_{\mathrm{t}}=n_{1}+n_{2}$. The round trip loss is determined by the refectivities, the coupler transmission and the typical splice transmission $T_{\mathrm{s}}$, experimentally measured through the cut back technique to be approximately $99 \%$. At threshold the gain is fixed by $R_{1} R_{2} T_{\mathrm{s}}{ }^{4} T_{\mathrm{c}}{ }^{2} G=1$, and the upper state population $\mathrm{N}_{2}$ is then determined by

$$
G=e^{\Gamma \int\left(n_{2}(x) \sigma_{e}-n_{1}(x) \sigma_{a}\right) d x}=e^{\frac{2 \Gamma}{A}\left(N_{2} \sigma_{e}-N_{1} \sigma_{a}\right)}
$$

Notably, $G$ is better expressed in terms of the total level populations, since the pumping and resulting populations are not uniform along the fiber length. The threshold is determined by finding the pump power at threshold, $P_{\mathrm{th}}$, required to balance the sum of the density dependent decay rates from the excited levels:

$$
\frac{(C R) P_{\text {th }}}{h v_{\text {pump }}}=\int\left(\gamma_{0}+2 k n_{2}\right) n_{2} d V=\gamma_{0} N_{2}+2 k \frac{N_{2}^{2}}{2 V}(1.2)=\frac{N_{2}}{\tau_{\text {eff }}}
$$

The standard definition for the thulium cross relaxation coefficient $\left(k=k_{1310}\right)$ has been used [4]. To simplify the expression, we used the fact that $n_{2}$ is small compared to $n_{1}$ and that $n_{2}$ is approximately determined by the local pump power $\left(n_{2}=n_{0} e^{-\alpha z}\right)$. The integration factor of 1.2 would be 1.0 in the limit of long fiber length compared to absorption length. The low density lifetime $\left(\tau_{0}=1 / \gamma_{0}\right)$ was measured by electrical pulse pumping and fluorescent monitoring of the decay at long time scales. For times greater than about $500 \mu \mathrm{s}$, the decay rate was a simple exponential and gave a lifetime of $480 \mu \mathrm{s}$. This is approximately consistent with the range and composition dependent values for the lifetime reported in the literature [17]. The parameters in Equations (3) and (4) that were not measured by us for this fiber were $\sigma_{\mathrm{e}}$ and $k$. To fit our measured values for threshold, $\sigma_{\mathrm{e}}$ has to be reduced by $40 \%$, assuming the fitted value of $k\left(1.5 \times 10^{-23} \mathrm{~m}^{3} \mathrm{~s}^{-1}\right)$ taken from [4] is correct for our fiber. This $k$ value gives an effective lifetime $\tau_{\text {eff }}$ of $380 \mu$ s for our fiber at threshold.

\subsection{Atomic Spectroscopy}

The above laser was designed for convenient optical quenching in an atomic spectroscopy experiment in helium [18]. The fiber laser operating at $2058.65 \mathrm{~nm}$ is used for quenching transitions in ${ }^{3} \mathrm{He}(2058.63 \mathrm{~nm})$ and ${ }^{4} \mathrm{He}(2058.69 \mathrm{~nm})$ singlets $\left(2^{1} \mathrm{~S}_{0}\right)$. The atomic beam of meta-stable helium is created by electron collisions and has an approximately room temperature thermal distributions of velocities ( $\mathrm{vavg}_{\mathrm{avg}} \approx 1500 \mathrm{~m} / \mathrm{s}$ ). The quenching is accomplished when the atoms cross the $\approx 3 \mathrm{~mm}$ diameter laser beam and undergo laser transitions to the fast decaying ${ }^{1} \mathrm{P}_{0}$ state (Figure 5). With high-power $(>0.5 \mathrm{~W})$, the broad emission spectrum is sufficiently stable and continuous to allow for efficient quenching of ${ }^{3} \mathrm{He}$ and ${ }^{4} \mathrm{He}$ simultaneously, insensitive to temperature and alignment fluctuations and drifts. At low power, the inefficient quenching of the states leads to sensitivity to the size of the beam, the power applied, and the transit time of atoms across the laser beam. Also, near threshold, the laser does not produce a uniform and stable frequency spectrum. Thus, the atomic quenching does not follow a simple exponential decay versus overall applied laser power, see Figure 5.

The bandwidth of the laser is an important design parameter which is approximately given by the FWHM of the output grating given sufficient high input pumping above threshold. When applied to the atomic beam, the laser can quench the singlet states down to a background level $(<1 \%)$ as shown (Separate experiments indicate the residual signal is from UV background photons) [19]. 


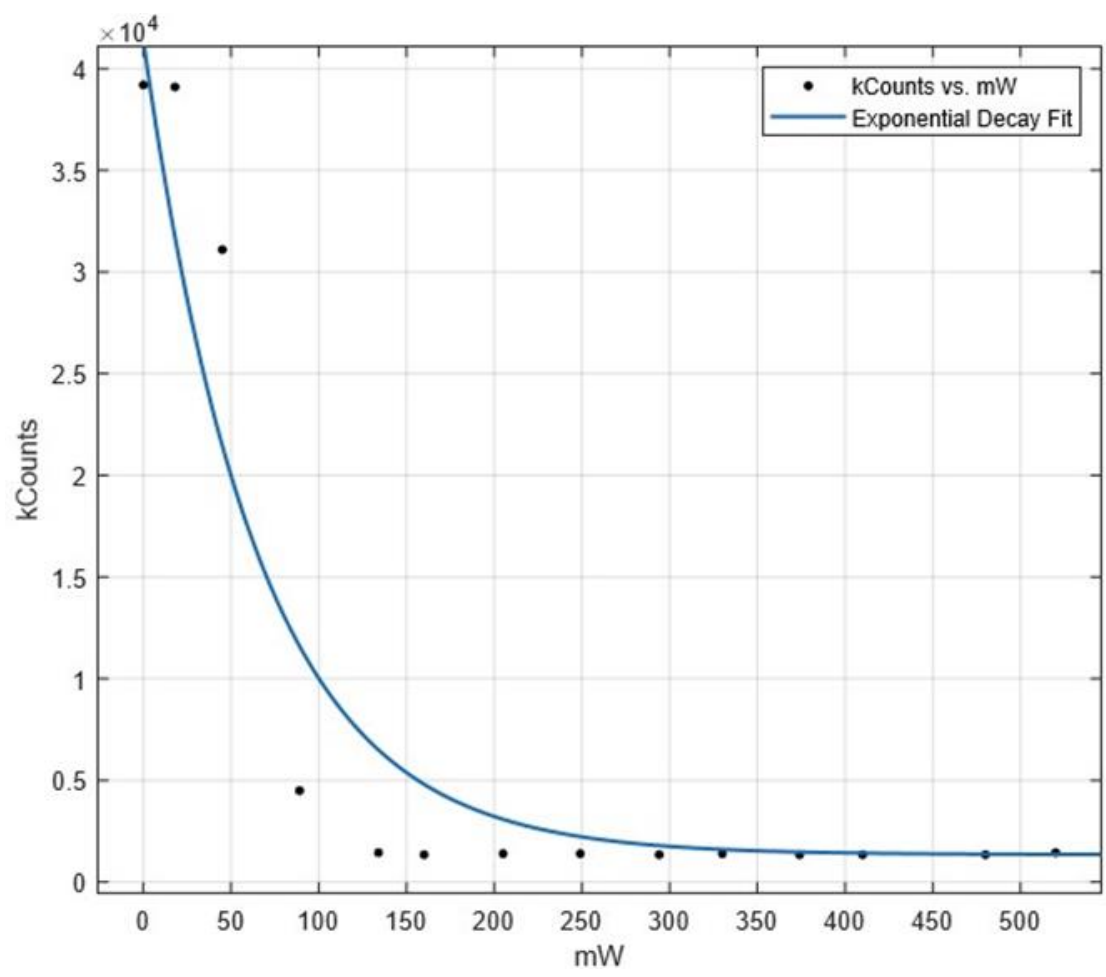

Figure 5. Quenching of the Singlet States.

\section{Discussion}

This paper describes the use of a thulium fiber laser operating at $2059 \mathrm{~nm}$ in an atomic spectroscopy experiment. The laser effectively quenches the singlet states of atomic helium, while being very insensitive to laboratory temperature and alignment changes, making it a very convenient and reliable solution. These results indicate that customized Tm double clad fiber lasers might provide effective solutions in similar optical pumping and spectroscopy applications. The simplified analysis and published cross-sections support readily feasible laser designs between 1900 and $2100 \mathrm{~nm}$. The paper shows a simple and effect fiber laser source that utilizes readily available materials to accomplish scientific experiments.

Author Contributions: This article was supported by D.S. through writing-review and editing, supervision, resources, and validation. A.K. contributed through validation and conceptualization. R.C. was responsible for writing-original draft preparation, formal analysis, data curation, methodology. All authors have read and agreed to the published version of the manuscript.

Funding: This research was supported by NSF award number 1404498.

Conflicts of Interest: The authors declare no conflict of interest.

\section{References}

1. Richardson, D.J.; Nilsson, J.; Clarkson, W.A. High power fiber lasers: Current status and future perspectives. J. Opt. Soc. Am. B 2010, 27, B63-B92. [CrossRef]

2. Zervas, M.N.; Codemard, C.A. High power fiber lasers: A review. IEEE J. Sel. Top. Quantum Electron. 2014, 20, 219-241. [CrossRef]

3. Moulton, P.F.; Rines, G.A.; Slobodtchikov, E.V.; Wall, K.F.; Frith, G.; Samson, B.; Carter, A.L.G. Tm-doped fiber lasers: Fundamentals and power scaling. IEEE J. Sel. Top. Quantum Electron. 2009, 15, 85-92. [CrossRef]

4. Jackson, S.D.; King, T.A. Theoretical modeling of tm-doped silica fiber lasers. J. Light. Technol. 1999, 17, 948-956. [CrossRef]

5. Hanna, D.; Percival, R.M.; Smart, R.G.; Tropper, A.C. Efficient and tunable operation of a tm-doped fibre laser. Opt. Commun. 1990, 75, 283-286. [CrossRef] 
6. Huang, Y.; Jivraj, J.; Zhou, J.; Ramjist, J.; Wong, R.; Gu, X.; Yang, V.X.D. Pulsed and cw adjustable $1942 \mathrm{~nm}$ single-mode all-fiber tm-doped fiber laser system for surgical laser soft tissue ablation applications. Opt. Express 2016, 24, 16674-16686. [CrossRef] [PubMed]

7. Jackson, S.D.; King, T.A. High-power diode-cladding-pumped tm-doped silica fiber laser. Opics Lett. 1998, 23, 1462-1464. [CrossRef] [PubMed]

8. Walbaum, T.; Heinzig, M.; Schreiber, T.; Eberhardt, R.; Tunnermann, A. Monolithic thulium fiber laser with $567 \mathrm{w}$ output power at $1970 \mathrm{~nm}$. Opt. Lett. 2016, 41, 2632-2635. [CrossRef] [PubMed]

9. Frith, G.; Carter, A.; Farroni, J.; Farley, K.; Tankala, K. Efficient and reliable 790nm-pumped Tm lasers from 1.91 to $2.13 \mu \mathrm{m}$. In Proceedings of the SSDLTR, Albuquerque, NM, USA, 4 June 2008.

10. Jackson, S. Cross relaxation and energy transfer up-conversion processes relevant to the functioning of $2 \mu \mathrm{m}$ $\mathrm{tm}^{3+}$-doped silica fibre lasers. Opt. Commun. 2004, 14, 197-203. [CrossRef]

11. Christensen, G.F.S.; Samson, B. Developments in thulium-doped fiber lasers offer higher powers. In Proceedings of the 21st Annual Meeting LEOS, IEEE Lasers Electro-Optics Society, Newport Beach, CA, USA, 9-13 November 2008.

12. Siegman, A. Lasers; Chapter 12; University Science Books: Mill Valley, CA, USA, 1986.

13. Walsh, B.; Barnes, N. Comparison of tm: Zblan and tm: Silica fiber lasers; spectroscopy and tunable pulsed laser operation around $1.9 \mu \mathrm{m}$. Appl. Phys. B 2004, 78, 325-333. [CrossRef]

14. Agger, S.D.; Povlsen, J.H. Emission and absorption cross section of thulium doped silica fibers. Opt. Express 2006, 14, 50-57. [CrossRef] [PubMed]

15. Romano, C.; Tench, R.; Jaouen, Y.; Williams, G. Simulation and design of a multistage 10w thulium-doped double clad silica fiber amplifier at $2050 \mathrm{~nm}$. In Fiber Lasers XIV: Technology and Systems; International Society for Optics and Photonics: San Francisco, CA, USA, 2017; p. 10083.

16. Peterka, P.; Kasik, I.; Dhar, A.; Dussardier, B.; Blanc, W. Theoretical modeling of fiber laser at $810 \mathrm{~nm}$ based on thulium-doped silica fibers with enhanced 3h4 level lifetime. Opt. Express 2011, 19, 2773-2781. [CrossRef] [PubMed]

17. Cajzl, J.; Paterka, P.; Kowalczyk, M.; Tarka, J.; Sobon, G.; Sotor, J.; Aubrecht, J.; Honzatko, P.; Kasik, I. Thuliumdoped silica fibers with enhanced fluorescence lifetime and their application in ultrafast fiber lasers. Fibers 2018, 6, 66. [CrossRef]

18. Smiciklas, M.; Shiner, D. Determination of the fine structure constant using helium fine structure. Phys. Rev. Lett. 2010, 105, 123001. [CrossRef] [PubMed]

19. Rezaeian, N.H. A Precise Few-Nucleon Size Difference by Isotope Shift Measurements of Helium; University of North Texas: Denton, TX, USA, 2015.

(C) 2020 by the authors. Licensee MDPI, Basel, Switzerland. This article is an open access article distributed under the terms and conditions of the Creative Commons Attribution (CC BY) license (http://creativecommons.org/licenses/by/4.0/). 